\title{
Minimum Acceptable Diet and Factor Associated with It Among Infant and Young Children Age 6-23 Months in North Shoa, Oromia Region, Ethiopia
}

\author{
Getu Gizaw ${ }^{1, ~ *, ~ G u d i n a ~ T e s f a y e ~}{ }^{2}$ \\ ${ }^{1}$ Department of Population and Family Health, Jimma University, Jimma, Ethiopia \\ ${ }^{2}$ Department of Medicine and Health, Jimma University, Jimma, Ethiopia
}

Email address:

gechgizaw21@gmail.com (G. Gizaw)

${ }^{*}$ Corresponding author

To cite this article:

Getu Gizaw, Gudina Tesfaye. Minimum Acceptable Diet and Factor Associated with It Among Infant and Young Children Age 6-23 Months in North Shoa, Oromia Region, Ethiopia. International Journal of Homeopathy \& Natural Medicines. Vol. 5, No. 1, 2019 , pp. 1-7. doi: $10.11648 /$ j.ijhnm.20190501.11

Received: January 24, 2019; Accepted: March 7, 2019; Published: March 21, 2019

\begin{abstract}
Appropriate complementary feeding Practice is essential in the first two years of life for satisfactory growth and development of children and for prevention of childhood illness. Insufficient quantities, frequency and inadequate quality of complementary foods have a detrimental effect on health and growth in this critical period. The aim of this study was to assess minimum dietary diversity and meal frequency practice and determinants among infant and young children age between 6 and 23 months in Shoa, Oromia Region, Ethiopia. Institution-based cross-sectional study was carried out to select 200 mothers/caregivers with 6-23 months of children reside in Sheno health center from July 25 to August 25, 2017. An interviewer-administered questionnaire was used to gather information on socio-demographic, child feeding practices and health-related characteristics. Data were entered to Epi-Data version 3.02 and transported to SPSS version 21 for further analysis. Binary logistic regression was used to see the association between the outcome variables and explanatory variables, and multivariable logistic regression was performed to identify independent predictors of minimum acceptable diet. The study revealed that the percentage of 6-23 months of children who meet the recommended level of minimum dietary diversity and meal frequency were 45 and 33\%, respectively. Proportion of children who received composite indictor minimum acceptable diet was only $13.3 \%$. Mothers/caregivers who had postnatal care visit, having good knowledge about child feeding practice, getting media exposure and mothers who had growth monitoring follow up were positively associated with minimum acceptable diet. Even though the study showed better progress as compared to the national prevalence of complementary feeding practices, child feeding practices in the study area were inadequate and not achieving national and WHO infant and young child feeding recommendations. Strengthening the available strategies and creating new intervention measures to improve maternal and child health services and giving behavioral change communication on child feeding practice using local media are compulsory actions for the government and policymakers.
\end{abstract}

Keywords: Dietary Diversity, Meal Frequency, 6-23 Months of Children, Northern Ethiopia

\section{Introduction}

Malnutrition is responsible, directly or indirectly, for over half of all childhood deaths. Infants and young children are at increased risk of malnutrition from six months of age onwards, when breast milk alone is no longer sufficient to meet all nutritional requirements and complementary feeding needs to be started [1].
Complementary feeding is the process starting when breast milk can no longer sufficiently meet the required nutritional for infants, and therefore introduction of other foods and liquids are needed, along with breast milk [2]. Complementary feeding generally begins at 6 months and goes up to 24 months of age, even though breastfeeding may continue beyond two years $[2,3]$.

The first 1000 days [time from conception up to 2 years of 
age] of the child's life provide a critical window of opportunity to ensure survival, growth and development through optimum infant and young child feeding (IYCF) practices [4] and inappropriate infant and young child feeding practices is significant threats to child health $[5,6]$.

The WHO therefore has recommended tracking IYCF indicators on the introduction of soft, solid or semi-solid foods, minimum dietary diversity, minimum meal frequency, and minimum acceptable diet during two years of age [7].

Children aged 6-23 months old should be given foods from 4 or more food groups out of the 7 recommended food groups namely: legumes and nuts; vitamin-A rich fruits and vegetables; other fruits and vegetables grains, roots and tubers; dairy products; flesh foods (meat, fish, poultry and organ meats); eggs; [7]. The cut-off of at least 4 of the above 7 food groups above was selected because it is associated with better quality diets for both breastfed and non-breastfed children [8]. The number of meals should be: 2 times for breastfed infants 6-8 months; 3 times for breastfed children 9-23 months and 4 times for non-breastfed children 6-23 months [7].

Global estimates for feeding of children aged 6 months to 2 years indicate substantial room for improvement. Only two thirds of 6-8 month olds are receiving any solid food at all, and when considering measures of diet quantity and quality, the rates are much lower: only 1 in 2 receives a minimum meal frequency and less than 1 in 3 minimum diet diversity. Together, when considering both minimum meal frequency and minimum diet diversity, only about 1 in 6 children are receiving a "minimally acceptable diet". In Ethiopia, 1 in 14 children receive minimally acceptable diet which is very poor figure [9]. Even though In the last few years in Ethiopia, some important advances in breastfeeding promotion have been made, but unfortunately, the same does not apply to complementary feeding this suggests assessing the determinant factor to national IYCF recommendations at specific region.

Ethiopia has a high burden of under-nutrition among young children. IYCF practices are to be monitored continuously to provide evidence-based decision-making in interventions designed to reduce under-nutrition in Ethiopia. Few previous studies have reported on the time of introduction of complementary feeding, meal frequency, meal diversity and acceptable diet. Determinants that have been associated with complementary feeding practices are household wealth status, exposure to media, maternal knowledge [10]. While most of the studies reported based on the national surveys [11], these national reports do not necessarily reflect the every diverse ethnic communities of Ethiopia. Additional information is needed to provide more evidence to monitor progress at the local level. The current study aimed to measure the prevalence of minimum acceptable diet and the factors associated with these infant feeding practices in North Shoa, Oromia Region, Ethiopia.

\section{Methods}

\subsection{Study Area, Study Design and Participants}

Institution-based cross-sectional study design was carried out in Sheno Health Centre, Sheno town from July 25 to August 25, 2017. The town is found in North Shoa, Oromia Region, Ethipia and $76 \mathrm{~km}$ far away from Addis Ababa.

Mothers or caregivers of children 6-23 months of age who reside in Sheno town were the source population, whereas mothers/caregivers of children 6-23 months who visited health center during our study period where considered as study population.

\subsection{Sample Size Determination}

The sample size was determined using single proportion population formula by assuming the proportion of introduction of complementary foods as $50.4 \%$ in western Ethiopia [12], 5\% as level of significance and 5\% as degree of precision and which was 368 . Since the total population less than 10,000 which are 436 approximating this number using the correction formula the final sample size was 200 mothers/caregivers with 6-23 months of children were included in the study.

\subsection{Data Collection and Measurements}

Data were collected using interviewer-administered validated questionnaire in a face-to-face manner from mothers/caregivers of children 6-23 months of age. The questionnaire consists of three parts: socio-demographic characteristics of households, maternal and child health related features and child feeding practices. Twenty-fourhour recall method and food frequency questionnaire were used to assess dietary diversity and meal frequency. Ten first degree in nursing holders as data collector and three second degree holders who had previous experiences as supervisor were involved in the survey.

Structured questionnaire partly adopted from WHO assessment tool for infant and young child feeding (IYCF) practices were used and translated into local language by fluent speakers and back translated to English to validate the consistency. Training was given for data collectors and supervisors on how to interview and maintaining the quality of data. Pre-test was done on $5 \%$ of the participants out of the selected areas. Then, the questionnaire was rechecked for its precision and consistency, and necessary modifications were incorporated before commencing the actual data collection. The supervisors and the investigatory were regularly monitored and checked the completeness of the data in daily bases.

\subsection{Operational Definition}

\subsubsection{Minimum Dietary Diversity}

Number of children 6-23 months of age who receive foods from four or more food groups during the previous day. The seven food groups used for tabulation of this indicator were as follows: cereals, roots and tubers; legumes and nuts; dairy 
products [milk, yoghurt and cheese]; flesh foods [meat, fish, poultry and liver/organ meats]; eggs; vitamin A-rich fruits and vegetables and other fruits and vegetables [7].

\subsubsection{Minimum Meal Frequency}

Proportion of breastfed and non-breastfed children 6-23 months of age, who receive solid, semi-solid or soft foods [but also including milk feeds for non-breastfed children] the minimum number of times or more. Breastfed infants of age 6-8 and 9-23 months should obtain a minimum of two or three meals with one to two snacks and three or four meals with one to two snacks per day, respectively. But nonbreastfed infants of age 6-23 months should receive milk products at least twice a day [7].

\subsubsection{Minimum Acceptable Diet}

Proportion of children 6-23 months of age who receive a minimum acceptable diet [apart from breast milk], it includes those children who have received both the minimum dietary diversity and the minimum meal frequency [7].

\subsection{Statistical Analysis}

Data were entered to Epi-Data 3.02 and exported, cleaned and analysed by SPSS version 21. Missed data were explored, and normality for continuous variables was checked. Dietary diversity score (DDS) was computed out of seven from seven food groups. Reliability of the tool was done, and Cronbach's Alpha value was 0.78. Household economic status was measured by constructing a wealth index through principal component analysis. The indicator variables used for wealth index construction that fulfil the requirement of factor analysis were used. Finally it was ranked in tertial (low, middle and high wealth index).

The data were presented in tables and figures by computing the percentages of minimum dietary diversity, meal frequency and acceptable diet. Binary logistic regression was done for minimum acceptable diet outcome variables of $\operatorname{MAD}(1=$ met minimum acceptable diet, $0=$ not fulfill minimum acceptable diet). The strength of associations and statistical significances between explanatory variables and outcome variables were expressed using OR and $95 \%$ of confidence interval, respectively. All variables in the binary logistic regression with $\mathrm{p}$ value $<0.25$ were moved to multivariable logistic regression and done using backward likelihood ratio to control the possible confounders and to identify predictors of the outcome variables. At this level, model fitness was checked which was Hosmer-Lemonshow as 0.67 and no multicollinearity. The variable was considered to be predictive for each outcome variable at $p$ value of less than 0.05 .

\subsection{Ethics}

Ethical clearance was obtained from Jimma University ethical review board and permission was obtained from the sheno Town Health Bureau. Written consent to participate in the study was secured before conducting the interview and discussion.

\section{Results}

\subsection{Socio-Demographic Characteristics}

A total of 200 infants and young children aged 6 to 23 months were enrolled along with their mothers. The mean age $( \pm \mathrm{SD})$ of the mothers was $28.2 \pm 6.2$ years. Majority, 172 $(86 \%)$, of the participants were Oromo by ethnicity and 175 (87.5\%) were Orthodox Christians'. One-fourth, 52 (26\%), of the mothers were unable to read and write. About two-third, $131(65.5 \%)$ of the mothers were housewives (Table 1).

Table 1. Parental Level Characteristic Of Children Aged 6- 23 Months Sheno Health Center North Shoa, Ethiopia 2017 [ N=200].

\begin{tabular}{llll}
\hline Variables & & Number & \% \\
\hline \multirow{2}{*}{ Residence of household } & Urban & 102 & 51 \\
& rural & 98 & 49 \\
& Orthodox & 175 & 87.5 \\
Religion & Muslim & 19 & 9.5 \\
& Protestant & 6 & 3 \\
& Illiterate & 52 & 26 \\
Educational status & $1-8$ & 79 & 39.5 \\
& $9-10$ & 41 & 20.5 \\
& Above 10 & 28 & 14 \\
& Housewives & 131 & 65.5 \\
Occupation of the & Government employed & 30 & 15 \\
mother & merchant & 39 & 19.5 \\
\hline
\end{tabular}

\subsection{Child, Household Economic and Information-Related Characteristics}

Of the total children, 107 (53.5\%) were female and majority, 173(86.5\%), were within age group of 9-23. Most of children $187(93.5 \%)$ start complementary feeding within the age group of 4-6 and almost all feed with bottle 195(97.5\%). More than one -third, 69(34.5\%) mothers were found in low wealth index and 157 (78.5\%) of mothers involve in income generating activities. Around two-third129 $(64.5 \%)$ of mothers didn't get any information regarding feeding practice and have poor knowledge in child feeding practice $130(65 \%)$ (Table 2).

\subsection{Child and Health Care Level Related Characteristics}

More than one-third of mothers have no ANC visit and more than half $117(57.5 \%)$ of the children were born in home. Regarding post natal care majority $163(81.5 \% 0$ children didn't get care (Table 2). 
Table 2. Child Feeding, Household Economic and Health Service Utilization Related Characteristic of Children Aged 6-23 Months Sheno Health Center North Shoa, Ethiopia, 2017 (N=200).

\begin{tabular}{|c|c|c|c|}
\hline variables & & no. & $\%$ \\
\hline \multirow{2}{*}{ Age of child } & 6-8 months & 27 & 3.5 \\
\hline & 9-23 months & 173 & 86.5 \\
\hline \multirow{2}{*}{ sex of child } & male & 93 & 46.5 \\
\hline & female & 107 & 53.5 \\
\hline \multirow{2}{*}{ ANC visit } & Yes & 126 & 63 \\
\hline & No & 74 & 37 \\
\hline \multirow{2}{*}{ Place of delivery } & Home & 117 & 58.5 \\
\hline & Health institute & 83 & 41.5 \\
\hline \multirow{2}{*}{ PNC visit } & Yes & 37 & 18.5 \\
\hline & No & 163 & 81.5 \\
\hline \multirow{3}{*}{ Involvement in income generation } & Yes & 157 & 78.5 \\
\hline & No & 43 & 21.5 \\
\hline & Low & 69 & 34.5 \\
\hline \multirow[t]{3}{*}{ Wealth status } & Middle & 60 & 30 \\
\hline & High & 71 & 35.5 \\
\hline & poor & 130 & 65 \\
\hline \multirow[t]{2}{*}{ Knowledge about feeding practice } & fair & 32 & 16 \\
\hline & good & 38 & 19 \\
\hline \multirow{2}{*}{ Age start complementary food } & $<4$ months & 13 & 6.5 \\
\hline & 4-6 months & 187 & 93.5 \\
\hline \multirow{2}{*}{ Feeding methods } & Bottle feeding & 195 & 97.5 \\
\hline & Cup feeding & 5 & 2.5 \\
\hline \multirow{2}{*}{ Access to information about feeding practice } & Yes & 71 & 35.5 \\
\hline & No & 129 & 64.5 \\
\hline
\end{tabular}

\subsection{Dietary Diversity and Meal Frequency Practices}

The proportion of children who received the recommended minimum dietary diversity and meal frequency was $45 \%$ and $33 \%$ respectively. Dairy products were provided to the greatest number of children [95\%], and none of the children were given vitamin A source food and other fruits and vegetables (Figure 1). finally factors that are associated with feeding practice was assessed by using a composite indictor called minimum acceptable diet, Because appropriate feeding of children 6-23 months is multidimensional, it is important to have a composite indicator that tracks the extent to which multiple dimensions of adequate child feeding are being met. The minimum acceptable diet indicator combines standards of dietary diversity and feeding frequency by breastfeeding status. In this study only $13.5 \%$ of children were given minimum acceptable diet (figure. 2).

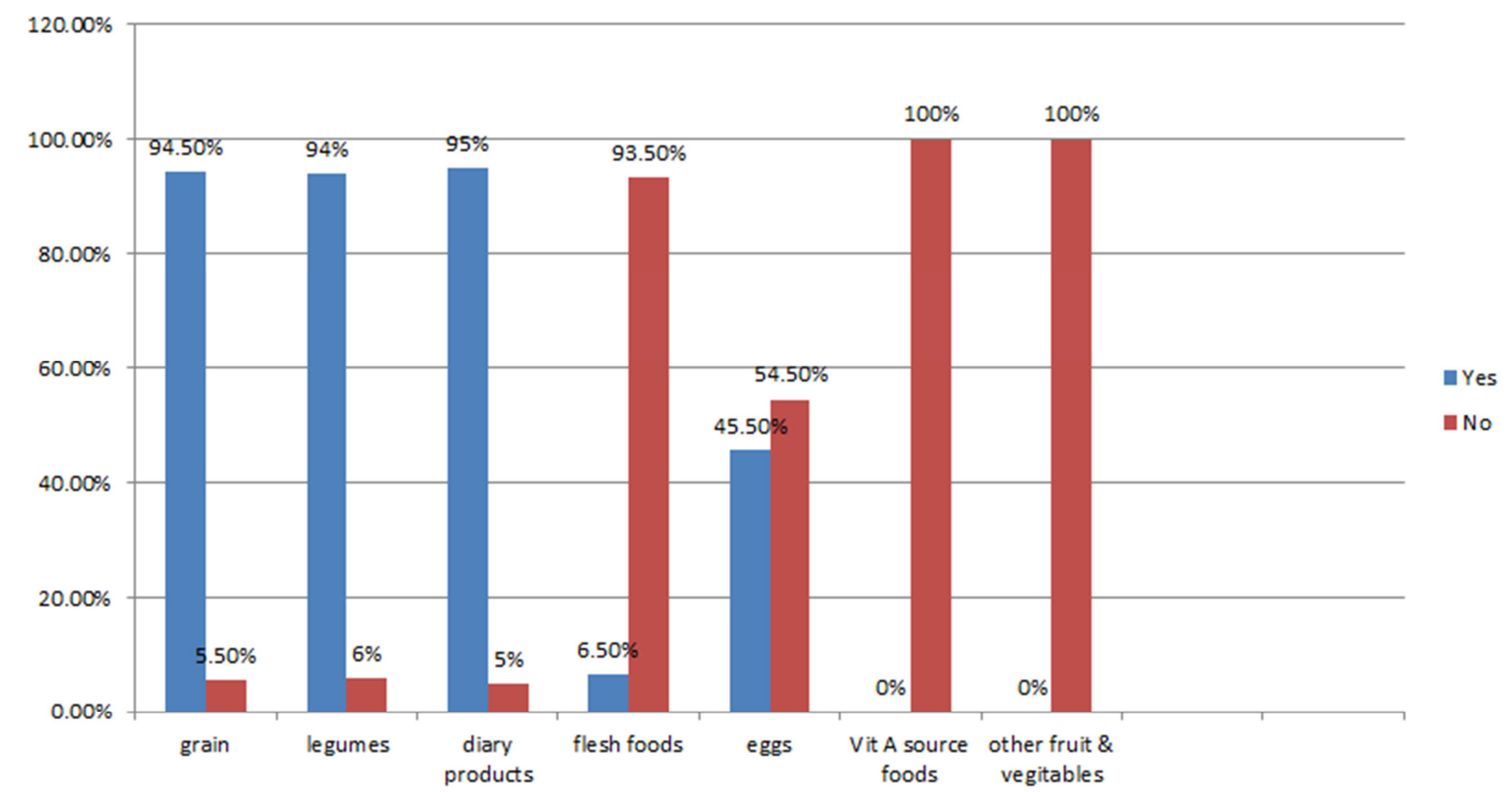

Figure 1. Types of food groups practiced among 6-23 months children, in sheno health center, North shoa, Ethiopia, 2017. 


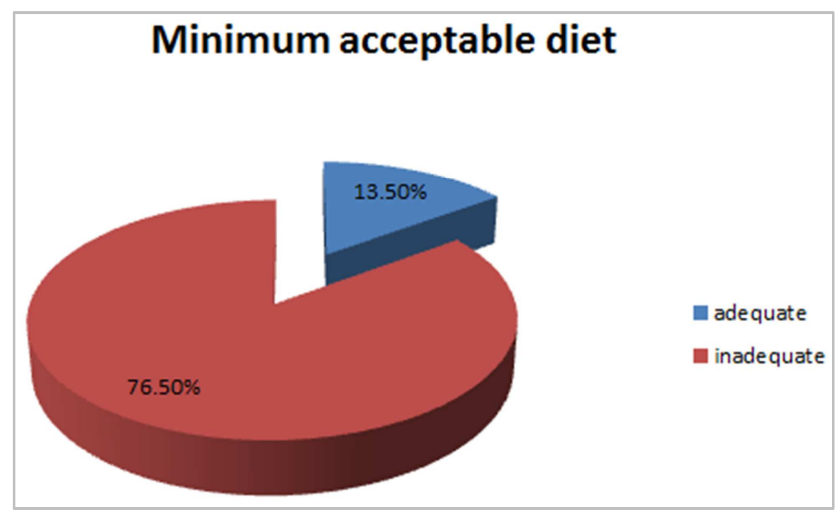

Figure 2. Intake of minimum acceptable diet among 6-23 months children, in sheno health center, North shoa, Ethiopia, 2017. 3.5. Factors Associated With Minimum Acceptable Diet.

Bivariate and multivariable logistic regression analysis was done using enter method to identify factors associated with Children receiving minimum acceptable diet. On the Bivariate analysis, Children minimum acceptable diet had statistical association with 10 variables which had $\mathrm{p}<0.25$.
On multivariable logistic regression analysis confirmed post natal care visit, child growth monitoring follow-up, access to information about child feeding practice and maternal knowledge on child feeding were potential predictor for Children minimum acceptable diet $(\mathrm{p}<0.05)$. (Table 3 ).

By making other variable constant mother participating in growth monitoring follow up were 5.3 times $[\mathrm{AOR}=5.3$, 95\% CI (1.43-19.65)] more likely to provide minimum acceptable diet to their child when compared to their counterparts. Similarly higher odds of receiving minimum acceptable diet was observed among mothers who had post natal care visits $[\mathrm{AOR}=4.4,95 \% \mathrm{CI}(1.22-16.05)]$.

Likewise, mothers who had access to information about child feeding practice were 5.3 times more likely to give recommended minimum acceptable diet compared to mothers who had no access $[\mathrm{AOR}=5.3$, 95\%CI (1.16-24.37)]. Finally, mothers who had good knowledge were 6 times more likely $[\mathrm{AOR}=6.15,95 \% \mathrm{CI}(1.61-23.490)]$ to give recommended minimum acceptable diet than mothers who had poor knowledge on child feeding practice.

Table 3. Multivariable Logistic Regression Table Predictor Of Minimum Acceptable Diet Among Children Aged 6-23 In In Sheno Health Center, North Shoa, Ethiopia, 2017.

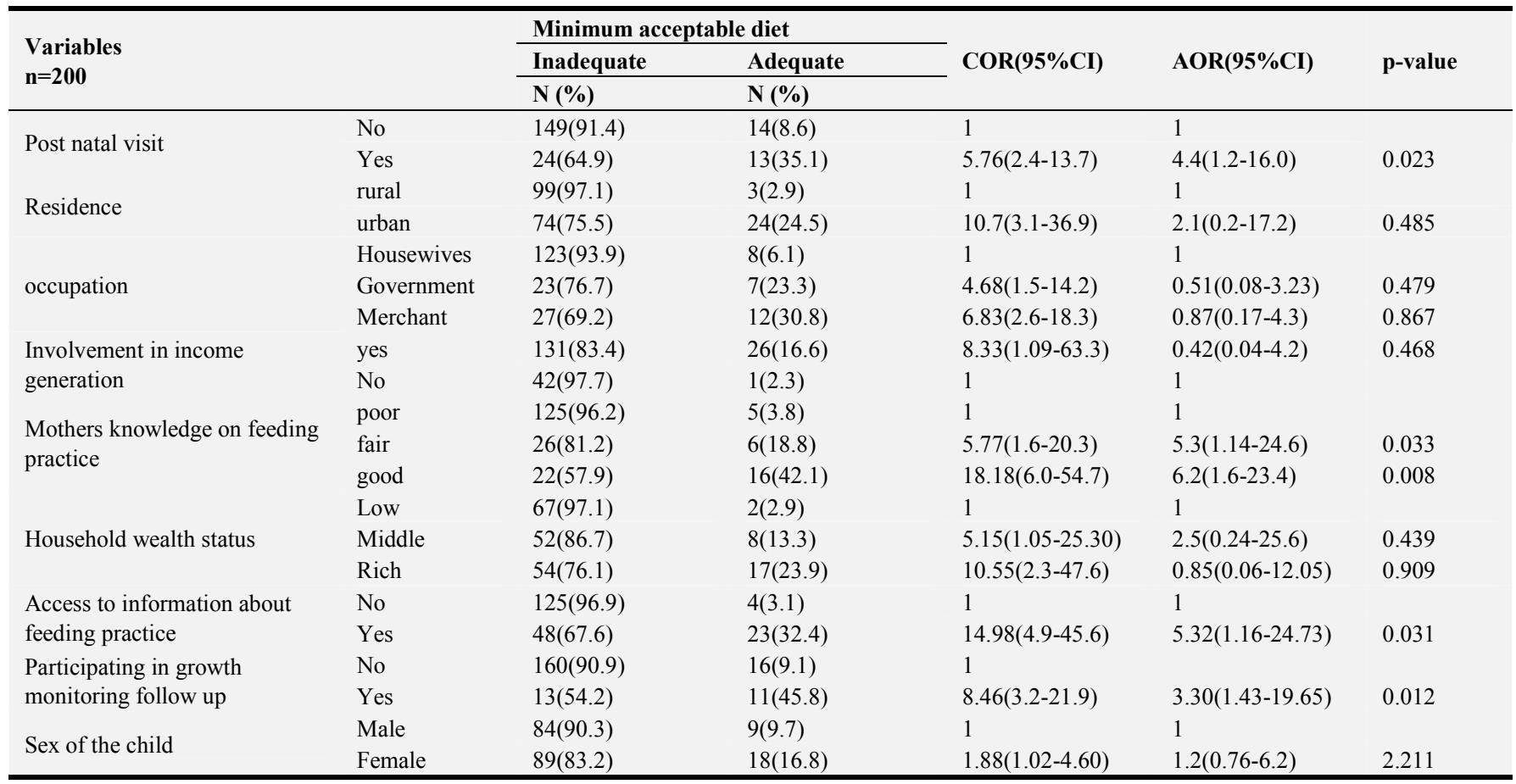

\section{Discussion}

This institution-based cross-sectional study identified that the proportion of children who received the minimum acceptable diet was $13.5 \%$. This finding is higher than the 2016 EDHS report of $7 \%$ [13]. This difference might be due to the fact that the EDHS was nationwide done on a larger sample. In addition, the EDHS was conducted on culturally different population, which may inhibit child feeding practices. While the current study was conducted on almost culturally homogenous population with similar feeding practices.

Determinants of receiving minimum acceptable diet in the study areas are multidimensional. Growth monitoring is conducted in every health facility according to the national nutrition program [14]. In these outreach clinics, rural health workers monitor the weight of children using growth charts, and provide nutrition education to mothers or caregivers of children. These activities also provide an opportunity to early recognition of signs of under nutrition, any illness and manage them accordingly. We found that such practices were positively associated with receiving minimum acceptable diet. 
The positive effect of such visits was also reported from other part of Ethiopia and also in Nepal $[15,16]$. so it should be strengthened growth monitoring program in study area and also at national level using health extension workers.

Mothers who had good knowledge about child feeding practice were about 6.2 times more likely to practice minimum acceptable diet compared to mothers with poor knowledge. This finding is in line with another finding in Uganda, Tehran and Indian [17-19]. Maternal knowledge and skill acquisition represents the maternal capacity to provide care.

Postnatal care visit was significantly associated with infant and young child feeding practices. Accordingly, mothers who had postnatal care visits were more likely to provide the recommended dietary diversity and meal frequency compared to their counterparts. This finding is supported by the finding from Sri Lanka [20]. This could be due to the fact that nutritional advice and counseling by health workers might not only educate mothers but also avoids traditional beliefs that might inhibit child feeding practices.

Mothers with satisfactory media exposure were about 2.8 times more likely to practice adequate dietary diversity compared to mothers with unsatisfactory media exposure. This finding is in line with another finding in Ethiopia [12, 15]. Because media promotion enhances timely, adequate, safe, and proper feeding practices. Moreover, the Ethiopian Ministry of Health and its partners distribute radio and family health cards to the health development army, which is an important tool for improving dietary diversity practices of the community.

\section{Conclusion}

The feeding practices of most mothers did not meet national and WHO recommendations. Behavior change communication about the importance of optimal complementary feeding, PNC and participation in child growth monitoring follow up should be strengthened through extensive use of the Health Extension Workers and media should focus on delivering message about child feeding practice to the community at large in the study area. It is recommended based on the result to the governments and stakeholders in the study area to make greater efforts to improve these critical practices in order to reduce child morbidity and mortality. Intervention studies on complementary feeding should target those health's seeking behavioral and media related factors that pose risks to optimal complementary feeding and to cut intergenerational cycle of malnutrition.

\section{Acknowledgements}

We would like to express their thankfulness to respective data collectors, supervisors and study participants for their diligence and devotion in the collecting and inputting High quality data used in the study.

\section{References}

[1] WHO. Guiding principles for complementary feeding of the breastfed child, Geneva, Switzerland. 2001.

[2] UNICEF. Infant and Young Child Feeding Programming Guide. 2011.

[3] Dewey KG, Brown KH. Update on technical issues concerning complementary feeding of young children in developing countries and implications for intervention programs. 2003; 24 [1]: 5-28.

[4] Robert E Black, Lindsay H Allen, Zulfiqar A Bhutta, Laura E Caulfield, Mercedes de Onis, Majid Ezzati, Colin Mathers JR. Maternal and child undernutrition: global and regional exposures and health consequences. 2004; 80: 131-41.

[5] Black RE, Allen LH, Bhutta ZA, Caulfield LE, Onis M De, Ezzati M, et al. Series Maternal and Child Undernutrition : global and regional exposures and health consequences. 2008; 5-22.

[6] UNICEF. Innocnti declaration 2005 on infant and young child feeding. 2015.

[7] WHO. Indicators for assessing infant and young child feeding practices. 2010

[8] USAID. Nutrition technical Developing and Validating Simple Indicators of Dietary Quality of Infants and Young Children in Developing Countries : Additional Analysis of 10 Data Sets September 2007 Working Group on Infant and Young Child Feeding Indicators, Wash. 2007.

[9] UNICEF. Child mortality estimates. 2017.

[10] Aemro M, Atnafu A, Meseie M, Birhanu Z. Dietary diversity and meal frequency practices among infant and young children age 6-23 months in Ethiopia: A secondary analysis of Ethiopian Demographic and Health. 2011.

[11] Central Statistical Agency. Ethiopia Demographic and Health Survey. 2011.

[12] Beyene M, Worku AG, Wassie MM. Dietary diversity, meal frequency and associated factors among infant and young children in Northwest Ethiopia Available from: http://dx.doi.org/10.1186/s12889-015-2333-x.

[13] Central Statistical Agency. Ethiopia Demographic and Health Survey. 2016.

[14] Strategy N. Ethiopia National Nutrition Strategy Review and Analysis of Progress and Gaps : One Year On. 2009.

[15] Belew AK, Ali BM, Abebe Z, Dachew BA. Dietary diversity and meal frequency among infant and young children: a community based study. Italian Journal of Pediatrics; 2017; 615 .

[16] Gautam KP, Adhikari M, Khatri RB, Devkota MD. Determinants of infant and young child feeding practices in Rupandehi , Nepal. BMC Res Notes. BioMed Central; 2016; $1-7$.

[17] Shams N, Mostafavi F, Hassanzadeh A. Determinants of complementary feeding practices among mothers of $6-24$ months failure to thrive children based on behavioral analysis phase of precede model, Tehran. 2016. 
[18] Ickes SB, Hurst TE, Flax VL. Maternal Literacy , Facility Birth, and Education Are Positively Associated with Better Infant and Young Child Feeding Practices and Nutritional Status among Ugandan Children 1 - 3. 2015; [6].

[19] Kuah JY, Manikam L. Systematic Review of Infant and Young Children Complementary Systematic Review of Infant and Young Children Complementary Feeding Practices [CFP] in South Asian Families. 2016; [September 2017].

[20] Senarath U, Dibley MJ, Kabir I, Khanam M, Agho KE, Mihrshahi S, et al. Complementary feeding practices in South Asia: Analyses by the South Asia Infant Feeding Research Network [SAIFRN]. 2012; 8 [January]. 